\title{
Rhesus D Antigenic Determinants on Residual Red Blood Cells in Apheresis and Buffy Coat Platelet Concentrates
}

\author{
Louis Thibault ${ }^{\mathrm{a}}$ Marie Joëlle de Grandmont ${ }^{\mathrm{a}} \quad$ Marie-Pierre Cayer $^{\mathrm{a}}$ \\ Nathalie Dussault $^{\mathrm{a}}$ Annie Jacques ${ }^{\mathrm{a}}$ Eric Ducas $^{\mathrm{a}}$ Annie Beauséjour ${ }^{\mathrm{a}}$ \\ André Lebrun ${ }^{b}$ \\ a Héma-Québec, Medical Affairs and Innovation, Québec, QC, Canada; \\ ${ }^{\text {b} H e ́ m a-Q u e ́ b e c, ~ M e d i c a l ~ A f f a i r s ~ a n d ~ I n n o v a t i o n, ~ M o n t r e ́ a l, ~ Q C, ~ C a n a d a ~}$
}

\section{Keywords}

Plateletpheresis · Platelet transfusion · Blood group antigen · Red cell contamination - RBC-derived particles · Alloimmunization

\begin{abstract}
Background: The level of residual red blood cells (RBCs) in platelet concentrates (PCs) is of interest because of clinical concerns related to alloimmunization to RBC antigens in transfused patients. This work aims at characterizing and quantifying the levels of intact and fragmented RBCs in apheresis (AP-PCs) and buffy coat PCs (BC-PCs) to assess their potential risk for $\mathrm{RhD}$ antigen alloimmunization. Methods: After staining with anti-CD41 (platelets) and antiCD235a (RBCs) antibodies, the size and density of RhD antigen on intact and fragmented RBCs were analyzed by flow cytometry. Results: Residual RBC counts were $29 \pm 22 \times 10^{6} \%$ unit in AP-PCs and $121 \pm 54 \times 10^{6} /$ unit in BC-PCs, which correspond to about 3 and $11 \mu \mathrm{L}$ of RBCs by product, respectively. RhD expression was about 4 times higher on RBC particles in AP-PCs, and these particles contribute to 66 and $75 \%$ of the total antigenic load in BC-PCs and AP-PCs, respectively. Conclusions: Processing methods influence the quantity and nature of contaminating residual RBCs and RBC-derived particles in PCs. The estimation of residual RBCs in these blood products is generally based on measurements of intact RBCs, which might underestimate the risk for alloim-
\end{abstract}

munization in transfused patients. The question of whether these RBC-derived particles can produce an immune response and, thus, should then be taken into consideration for Rh immune prophylactic treatments, remains to be clarified.

(c) 2019 S. Karger AG, Basel

\section{Introduction}

Platelets do not express the $\mathrm{RhD}$ antigen but the presence of contaminating residual red blood cells (RBCs) in platelet concentrates (PCs) from RhD-positive blood donors might trigger the generation of anti-D in $\mathrm{RhD}-$ negative transfused patients [1-4]. Despite the fact that the frequency of alloimmunization is low, RhD alloimmunization has been reported in non-immunocompromised recipients following injection of as little as $30-50 \mu \mathrm{L}$ of RBCs [1, 5-9]. In Canada and in many other countries, transfusion of PCs from $\mathrm{RhD}$-positive donors to $\mathrm{RhD}$ negative recipients is avoided whenever possible, especially for female patients of childbearing age. For these patients, the choice of an Rh immune-prophylactic treatment is often highly recommended [4].

PCs can be obtained either by AP (AP-PCs) from single donors or by pooling 4-6 buffy coat units (BC-PCs), or platelet-rich plasma PCs obtained by whole blood centrifugation. At Héma-Québec, approximately $90 \%$ of PCs 
are obtained by single-donor AP, with the remainder of the transfusion needs being filled by semiautomatic processing of $\mathrm{BC}$ pools from 5 donors with the automated Atreus/OrbiSac system (TerumoBCT, Lakewood, CA, USA). Both PC types are considered clinically equivalent and meet Canadian regulatory standards for $\mathrm{pH}$, sterility, residual white blood cells, volume, and platelet content. However, some differences exist between these PCs regarding their residual RBC content, which could represent a potential risk for alloimmunization against RBC antigens in transfused patients [3, 4, 9-15]. Studies that investigated the impact of processing methods on the content of residual RBCs in PCs have shown considerably lower contaminating RBCs in AP-PCs $(0.17-9 \mu \mathrm{L})$ than in $\mathrm{BC}$-PCs $(36-590 \mu \mathrm{L})[3,8,10-12]$. In order to assist our partner transfusion centers in their $\mathrm{RhD}$ alloimmunization prophylaxis approach, and to assess the impacts of processing methods on contaminating RBCs in PCs, we have investigated residual RBCs in 150 PCs prepared by AP (Trima Accel, TerumoBCT) or by semiautomated BC pooling (Atreus/OrbiSac system, TerumoBCT) using a quantitative flow-cytometric method. Furthermore, the $\mathrm{RhD}$ antigenic burden and the membrane phospholipid asymmetry were also investigated.

\section{Methods}

\section{Blood Components}

Whole blood $(460 \mathrm{~mL})$ was collected from healthy donors following standard operation procedures. Blood was collected in 63-mL citrate-phosphate-dextrose anticoagulant in Atreus system collection sets (TerumoBCT). Immediately after collection, whole blood bags were rapidly chilled and stored at $20-24^{\circ} \mathrm{C}$ using Phase 22 phase change material [13]. Blood units were processed within $24 \mathrm{~h}$ into BCs, RBC concentrates, and plasma units with Atreus automates (3C protocol) according to the manufacturer's instructions (TerumoBCT). After a rest period, pools of $5 \mathrm{BCs}$ were prepared, leukoreduced by in-line filtration, and processed into final products using OrbiSac devices (TerumoBCT). Single-donor APPCs were collected at permanent blood centers with TRIMA Accel collection instruments (TerumoBCT). All platelet concentrates were stored in plasma.

Samples were obtained from AP-PCs and BC-PCs on days 1 or 2 after whole blood/AP collection, and products were put into the inventory by the quality control laboratory, as part of routine testing, and held at room temperature. Hematological and flow-cytometric measurements were completed on the same day. Platelet counts were determined using a Coulter AcT5diff AL Hematology analyzer (Beckman Coulter Canada, ON, Canada).

\section{Flow-Cytometric Analysis}

Analysis of CD235a-Positive Events in PCs. The analysis of residual RBCs and RBC-derived particles (collectively referred to as CD235a-positive events) was performed by flow cytometry, based on previous work $[14,15]$. Briefly, aliquots of PCs were diluted in PBS, transferred to a Trucount Tube (Becton Dickinson Biosciences, San Jose, CA, USA), and mixed with FITC-conjugated CD41a (clone HIP8) to label platelets and phycoerythrin (PE)conjugated CD235a (clone GA-R2) for RBCs (Becton Dickinson
A

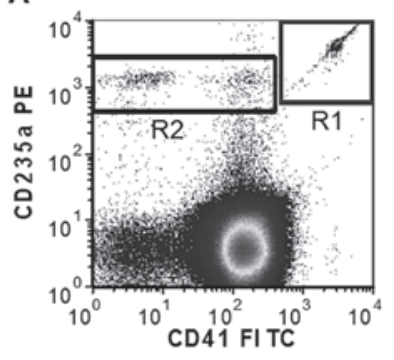

B

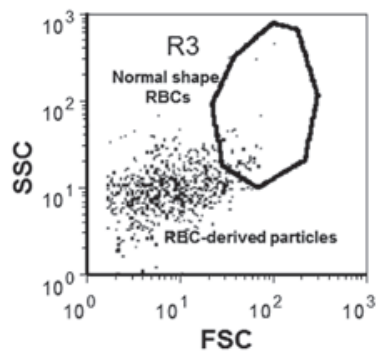

Fig. 1. Flow-cytometric gating strategy for the analysis of residual intact $\mathrm{RBCs}$ and $\mathrm{RBC}$-derived particles. Combined regions were used in a two-level gating strategy to characterize intact RBCs (CD235a-positive events) and RBC-derived particles. A Gate R1 encompassed Trucount ${ }^{\mathrm{TM}}$ beads, while gate $\mathrm{R} 2$ contained all CD235a-postive events associated or not with platelets (CD41positive events). B CD235a-positive events in gate $\mathrm{R} 2$ were further discriminated based on their differences in forward scatter (FSC) and side scatter (SSC) using gate R3. This gate has been determined using fresh RBCs, so all CD235a-positive events located outside of this gate were assumed to be RBC-derived particles.

Biosciences). After a 20 -min incubation at $20-24^{\circ} \mathrm{C}$ in the dark, samples were diluted in PBS and analyzed on either a CyFlow ML flow cytometer using FlowMax 3.0 software (Partec, Münster, Germany) or on an Accuri C6 flow cytometer using CFlow Plus software (Becton Dickinson, Ann Arbor, MI, USA) until 3,000 events (beads; corresponding to about $60 \mu \mathrm{L}$ ) were acquired in the R1 gate (Fig. 1A). Data were further processed using the FCS Express Flow research edition software (De Novo Software, Los Angeles, CA, USA). The limits of positive events for the CD41a and CD235a markers were established using FMO (fluorescence minus one) controls and the use of unstained (negative) samples [16]. Compensation was done using Compbeads (BD Biosciences). The R2 gate was established with PC samples spiked with intact RBCs stained with PE-conjugated CD235a antibody. This gate gathers all CD235a-positive events including intact, damaged, as well as fragmented RBCs, whether associated or not with platelets (CD235apositive/CD41a-positive events). CD235a-positive events were further reanalyzed according to their size (FSC) and granularity (SSC) and compared to fresh and unstained RBCs to segregate intact RBCs (R3 gate) from fragmented RBCs (Fig. 1B).

The linearity range of this assay was established between 300 and $10,000 \mathrm{RBCs} / \mu \mathrm{L}\left(R^{2}=0.9980\right)$ using PC samples spiked with known concentrations of RBCs. The limit of detection was established at $126 \mathrm{RBCs} / \mu \mathrm{L}$ with a coefficient of variation lower than $10 \%$. Moreover, this assay was comparable to Neubauer chamber manual counting of PC samples spiked with known concentrations of fresh RBCs $\left(R^{2}=0.9970\right)$ within the linear range of the assay.

Size Determination, Membrane Phospholipid Asymmetry, and RhD Expression on CD235a-Positive Events. The size of CD235apositive events in $\mathrm{R} 2$ and $\mathrm{R} 3$ gates was estimated using a standard curve of the FSC median fluorescence intensity of calibrated beads diluted in PBS and ranging in size from 0.3 to $15 \mu \mathrm{m}$ (Invitrogen Corp., Eugene, OR, USA). For these determinations, data have been acquired in triplicates. Size estimation of CD235a-positive events was performed on AP-PCs and BC-PCs ( $n=10$ each). The relationship between particle size and peak channel number of 
Table 1. Comparison of residual intact $\mathrm{RBCs}$ and $\mathrm{RBC}$-derived particle contents of BC-PCs $(n=150)$ and AP-PCs $(n=150)$

\begin{tabular}{lccc}
\hline & BC-PCs & AP-PCs & $p$ value \\
\hline Volume, $\mathrm{mL}$ & $349 \pm 16$ & $223 \pm 17$ & $<0.0001$ \\
Platelets, $\times 10^{11} /$ unit & $4.0 \pm 0.6$ & $3.5 \pm 0.4$ & $<0.0001$ \\
Residual $\mathrm{RBCs}, \times 10^{6} /$ unit $^{1}$ & $121 \pm 54$ & $29 \pm 22$ & $<0.0001$ \\
$\mu \mathrm{L} /$ unit $^{1}$ & $11 \pm 5$ & $3 \pm 2$ & $<0.0001$ \\
\hline RBC particles, $\times 10^{6} /$ unit $^{2}$ & $498 \pm 269$ & $420 \pm 166$ & 0.0028 \\
\hline
\end{tabular}

${ }^{1}$ The volume of residual RBCs per unit has been estimated based on a mean RBC volume of $90 \mathrm{fL}$. Values are presented as means $\pm \mathrm{SD}$.

FSC calibrated beads has been established by applying a three-term polynomial calculation as follows:

$$
y=4 \times 10^{-19} x^{3}-3 \times 10^{-12} x^{2}+8 \times 10^{-06} x+0.9277,
$$

where $x$ is the diameter in micrometers and $y$ is the equivalent channel number (size). The quantitative limit of detection was established at $1 \mu \mathrm{m}$ on our flow cytometers.

The membrane phospholipid asymmetry of residual intact $\mathrm{RBCs}$ and RBC-derived particles was also investigated with the same gating strategy using FMO and compensation adjustments with Alexa Fluor 488-conjugated annexin V antibody (Molecular Probes, Eugene, OR, USA) on 10 PCs of both types.

The quantification of $\mathrm{RhD}$ antigen expressed on CD235a-positive events (R2 gate) was estimated using the PE fluorescence quantitation kit QuantiBrite PE beads (Becton Dickinson Biosciences) according to the manufacturer's instructions on 10 PCs. Beads with various fluorescence intensity levels in the PE channel (FL2A) and defined numbers of surface PE molecules were used as standard. Prior to analysis, RBCs were washed 3 times by centrifugation $(1,000 \mathrm{~g}, 1 \mathrm{~min})$ with $0.9 \%$ saline and treated for $15 \mathrm{~min}$ at $37^{\circ} \mathrm{C}$ with $0.75 \%$ ficin to facilitate the labeling of $\mathrm{D}$ antigen. RBCs were next analyzed according to the same method described above for the analysis of CD235a events by adding PE-conjugated antiRhD antibody (Millipore, Billerica, MA, USA), assuming a PE: antibody ratio of $1: 1$. The specificity of this anti-D in flow cytometry was confirmed by the analysis of $\mathrm{RhD}$-positive and $\mathrm{RhD}$-negative RBCs. The amount of anti-D was adjusted to completely saturate the $\mathrm{D}$ antigen sites expressed on fresh RBCs of $\mathrm{RhD}$-positive individuals while preventing their agglutination.

\section{Statistics}

Values are presented as means \pm SD. Differences between BCPC and AP-PC groups were compared using Student's $t$ test for independent samples while the normality of data was estimated beforehand using the Kolmogorov-Smirnov test. All tests were two sided, and significance was reported at the $p<0.05$ level. Statistical analyses were performed using SAS 9.4 software (SAS Institute Inc., Cary, NC, USA).

\section{Results}

The quantitative analysis of residual RBCs and RBCderived particles was performed on $150 \mathrm{BC}$-PCs and an equal number of AP-PCs selected for this study. All these
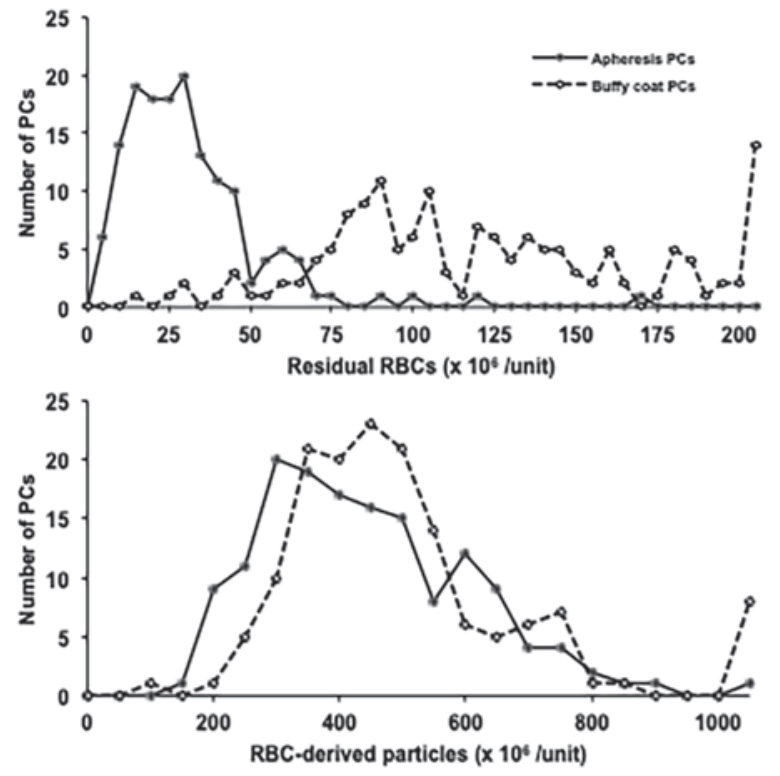

Fig. 2. Influence of the manufacturing process on the distribution of residual RBCs and RBC-derived particles in AP-PCs $(n=150)$ and BC-PCs $(n=150)$.

randomly selected PCs met Canadian regulatory standards for transfusion in terms of volume, platelet count, residual leukocytes, and sterility. The volume of BC-PCs was, on average, 1.5 times higher than AP-PCs with a comparable total platelet content (Table 1).

The analysis of CD235a-positive events, isolated in the R2 gate according to their size (FSC) and granularity (SSC), allows the segregation of intact RBCs (R3 gate) from $\mathrm{RBC}$-derived fragments or particles (Fig. 1). The method of PC manufacturing has an impact on the amount of residual RBCs found in these components (Table 1; Fig. 2). Indeed, AP-PCs contain on average 4.2 less normal size RBCs than BC-PCs $\left(29 \pm 22 \times 10^{6} \mathrm{RBCs} /\right.$ unit or $3 \pm 2 \mu \mathrm{L} /$ unit vs. $121 \pm 54 \times 10^{6} \mathrm{RBC} /$ unit or $11 \pm 5 \mu \mathrm{L} / \mathrm{unit} ; p<0.0001)$. For AP-PCs, this amount of residual RBCs is comparable to that of $27 \times 10^{6} /$ unit reported by Santana and Dumont [15]. The amount of contaminating intact $\mathrm{RBCs}$ is $2-5$ times lower than reported in manually prepared BC-PCs or platelet-rich plasma PC $[3,4,9,17,18]$. On the other hand, the total burden of RBC-derived particles per unit is similar for both types of PCs, despite a higher concentration in AP-PCs $(1,880 \pm$ 721 vs. $1,430 \pm 782 \mathrm{CD} 235$ a-positive events/ $\mu \mathrm{L} ; p<0.001)$. Figure 2 shows distribution curves of the number or residual RBCs (Fig. 2A) and RBC-derived particles (Fig. 2B), for both AP-PCs and BC-PCs.

The average size of RBC-derived particles in AP-PCs is smaller than that of BC-PCs $(1.7 \pm 0.2 \mu \mathrm{m}$ vs. $3.1 \pm$ 
Table 2. Estimation of RhD antigen density on residual RBCs and RBCderived particles found in single-donor AP-PCs and BC-PCs $(n=10)$

\begin{tabular}{lccr}
\hline & BC-PCs & AP-PCs & $p$ value \\
\hline Residual RBCs & & & \\
$\quad$ Size, $\mu \mathrm{m}$ & $5.9 \pm 0.4$ & $6.2 \pm 1.2$ & 0.3399 \\
Level of RhD antigen/cell & $3,721 \pm 990$ & $15,319 \pm 8,750$ & 0.0006 \\
Density of RhD antigen/ $\mu \mathrm{m}^{2}$ & $34 \pm 8$ & $125 \pm 57$ & $<0.0001$ \\
Annexin V+ cells, \% & $10 \pm 6$ & $73 \pm 21$ & $<0.0001$ \\
RBC-derived particles & & & \\
Size, $\mu \mathrm{m}$ & $3.1 \pm 0.3$ & $1.7 \pm 0.2$ & $<0.0001$ \\
Level of RhD antigen/particle & $1,759 \pm 493$ & $3,077 \pm 2,314$ & 0.0952 \\
Density of RhD antigen/ $\mu \mathrm{m}^{2}$ & $56 \pm 10$ & $326 \pm 176$ & 0.0001 \\
Annexin V+ particles, $\%$ & $16 \pm 7$ & $58 \pm 9$ & $<0.0001$ \\
\hline
\end{tabular}

The density of RhD determinants on RBC particles was estimated assuming a spherical shape, based on their mean size in $\mu \mathrm{m}$ and the surface area of a sphere given by the following formula: $4 \pi r^{2}$. Values are presented as means \pm SD.
$0.3 \mu \mathrm{m}$, respectively; $p<0.0001$ ) (Table 2 ). As expected, the size of residual RBCs, identified as CD235a-positive events in R3 gate, corresponds to the size of fresh RBCs. Further characterization of residual RBCs and RBC-derived particles in terms of $\mathrm{RhD}$ antigen content was next performed to investigate their potential to produce an immune reaction in patients (Table 2). The manufacturing method appears to influence the expression of the $\mathrm{D}$ antigen on residual RBCs and RBC-derived particles. RBCs found in single-donor AP-PCs (gate R3) seem less affected by the collection procedure, carrying an antigenic density comparable to that of fresh $\mathrm{RhD}$-positive $\mathrm{RBCs}$, i.e., $15,319 \pm 8,750$ sites per cell [19]. In addition, nearly $73 \%$ of these residual cells are eryptotic (annexin $\mathrm{V}$ positive) compared to BC-PCs which express about 4 times less $\mathrm{RhD}$ sites and only $10 \%$ eryptotic residual cells.

The flow-cytometric method used in our study allowed us to further characterize the immune potential of RBC debris present in PCs that are generally disregarded when quantifying contaminating RBCs in PCs. The results of RhD-determinant quantification, presented in Table 2, indicate that these RBC particles clearly express $\mathrm{D}$ antigen epitopes on their surface at a density lower than that measured on residual intact RBCs found in the same PC units. In fact, these RBC particles contribute to 66 and $75 \%$ of the total $\mathrm{RhD}$ antigenic load in PCs derived from whole blood and AP, respectively.

\section{Discussion}

The presence of RBCs in platelet concentrates remains a transfusion concern because of their potential to generate an allogeneic response in some patients. This work reports, like other studies, a low level of RBCs in platelet concentrates and highlights the influence of the processing method $[3,4,8,20]$. Alloimmunization to red cell an- tigens following PC transfusion is rather uncommon and has substantially decreased over the years [6]. In earlier reports, incidences of $\mathrm{RhD}$ alloimmunization as high as $19 \%$ have been reported in $\mathrm{RhD}$-negative patients receiving PC transfusions from $\mathrm{RhD}$-positive donors [17]. In a 10-year retrospective study, Cid et al. $[3,4,8]$ observed an alloimmunization rate ranging from 0 to $7 \%$ in $\mathrm{RhD}$ negative patients transfused with $\mathrm{RhD}$-positive PCs. The lower incidence observed over the years is linked to technological advances in the AP equipment as well as the automation of whole blood processing for PC production [2]. The likelihood of developing anti-RhD antibodies is higher than for any other blood group antigen. Indeed, following $\mathrm{RhD}$-incompatible $\mathrm{RBC}$ concentrate transfusion, the rate of antigen alloimmunization has been estimated at about $30 \%$ in $\mathrm{RhD}$-negative patients, as high as $47 \%$ in sickle cell patients, and $28-37 \%$ in thalassemia patients [21-24]. Additionally, $\mathrm{RhD}$ alloimmunization has been reported in nonimmunocompromised recipients following repeated injections of as little as $30 \mu \mathrm{L}$ of RBCs $[5,6]$. RBC antigen alloimmunization is a multifactorial adverse transfusion reaction influenced by the recipient's immune competence and inflammation state, the nature and density of the antigens, and the number and type of infused blood products [2, 24-26]. Why some transfused patients develop an anti-RBC antigen antibody while others do not is still not well understood, but for those who have already developed anti- $\mathrm{D}$, the transfusion of antigen-negative blood products to prevent adverse hemolytic transfusion reactions can become a major challenge for blood banks.

Although the measurement of residual RBC content in PCs could qualify as a quality control indicator, there is, currently, no regulatory requirement in Canada for residual RBC content in PCs, and only products deemed "red" by the blood bank staff are routinely removed from the inventories. In this work, we have developed a simple 
flow-cytometric method to assess the amount of residual RBCs. This method offers the possibility of quantifying not only intact contaminating RBCs but also of obtaining information on the RBC antigenic burden both qualitatively and quantitatively. This method is accurate, reproducible, and can be easily adapted to the equipment already in use in most blood bank quality control laboratories. The results presented in this paper also highlight the influence of the PC processing method on residual RBC measurements made in these products. Furthermore, residual RBC content in PCs generally refers to the observation of cells having a size corresponding to that of fresh and intact RBCs. In this study, we show that the level of residual $\mathrm{RBC}$ contaminants in PCs are lower in AP products than in $\mathrm{BC}$ pools, suggesting a lower risk of alloimmunization to erythrocyte antigens with AP-derived products.

The preservation of the number of $\mathrm{RhD}$ antigenic sites on residual intact $\mathrm{RBC}$ s observed in AP-PCs suggests that these cells are less affected by the AP procedure. However, these contaminating RBCs are significantly more eryptotic than those found in BC-PCs (73 vs. $10 \%$ ) suggesting that residual RBCs in AP-PCs are likely to be more rapidly removed from the circulation following transfusion $[4,27]$. RhD-positive events of intact size RBCs observed in BC-PCs have a slightly smaller size and express, on average, 4 times less $\mathrm{RhD}$ determinants than fresh and intact RBCs. Since the $\mathrm{RhD}$ antigen consists of a set of conformational epitopes [28], this decline in the number of $\mathrm{RhD}$ antigens could be attributed to membrane or cytoskeleton damage or to alterations in the antigenic epitopes recognized by the monoclonal anti-D used. The lower $\mathrm{RhD}$ expression and percent of eryptotic cells in BC-PCs might seem contradictory. Compared to the AP procedure, the production of $\mathrm{PCs}$ by the $\mathrm{BC}$ method is semiautomated and requires additional steps (e.g., storage time, centrifugation, pooling, transfer, and leukoreduction) that might have further affected $\mathrm{RBC}$ integrity in BC-PC without necessarily triggering eryptosis. Conversely, the residual RBCs in AP-PCs would be more "intact" and close to what is found in the circulation and might already have entered eryptosis.

$\mathrm{RBC}$ particles could potentially be as immunogenic as intact RBCs, especially since they can be more easily phagocytosed by antigen-presenting cells of the recipient $[4,29,30]$. Moreover, these fragmented RBCs, once released into the circulation, could lead to inflammation or posttransfusion thrombosis and could also represent a potential risk for RBC alloimmunization in transfused patients $[1,18,29-36]$. Our attempt at measuring the size of RBC particles in AP-PCs and BC-PCs was, however, limited by our flow-cytometric instruments, which prevented us from accurately measuring particle sizes $<1 \mu \mathrm{m}$. CD235a-positive particles encompass both RBC-derived microparticles $(\leq 1 \mu \mathrm{m})$ and larger $\mathrm{RBC}$ particles, as previously described by Kitazawa et al. [1]. Compared to residual RBCs, RBC-derived particles express about $75 \%$ of the total RhD antigen burden in AP-PCs and about $66 \%$ of the D antigen load in BC-PCs. The question remains, however, whether these particles represent an additional risk for alloimmunization for antigen-negative patients [1]. We acknowledge that our study was limited not only by the small number of observations, but also by the very low level of contaminating cells in PCs, the studied PC processing methods, and the analysis limited to the $\mathrm{RhD}$ antigen. However, we hope that this work can stimulate other studies aimed at mitigating the adverse effects of alloimmunization associated with PC transfusion.

In conclusion, this study shows that processing methods influence the nature and quantity of contaminating residual RBCs and RBC-derived particles in PCs. By quantifying $\mathrm{RhD}$ antigenic determinants, we show that RBC particles actually express a substantial amount of antigenic determinants, which underscores the substantial contribution of RBC-derived particles to the immune antigenic pool in PCs [4, 9]. Whether RBC-derived particles can contribute, along with residual intact $\mathrm{RBC}$, to the induction of an alloimmune response in transfused recipients, and whether antigen-carrying particles should be taken into consideration for $\mathrm{RhD}$ immune prophylactic treatments, remains to be investigated.

\section{Acknowledgments}

We would like to thank our colleagues from the quality control laboratory for their assistance with the analysis of platelet concentrates as well as Dr. Josée Aubin for her assistance with the drafting of the manuscript. We are also grateful to Antoine Lewin for his statistical analyses and to Dr. Sonia Néron as well as Jean-Francois Leblanc for their critical review and comments.

\section{Statement of Ethics}

All donors have signed the standard informed consent form for either whole blood donation or AP platelet collection, as applicable.

\section{Disclosure Statement}

The authors have no conflict of interests to declare.

\section{Author Contributions}

All authors participated to the design of this study. L.T. drafted the manuscript. M.J.G., M.-P.C., N.D., E.D., A.B., and A.L. critically reviewed the manuscript. All authors approved the final version. 


\section{References}

1 Kitazawa J, Nollet K, Morioka H, Tanaka K, Inomata M, Kubuki Y, et al. Non-D Rh antibodies appearing after apheresis platelet transfusion: stimulation by red cells or microparticles? Vox Sang. 2011 May;100(4):395400.

2 Shaz BH, Hillyer CD. Residual risk of D alloimmunization: is it time to feel safe about platelets from D+ donors? Transfusion. 2011 Jun;51(6):1132-5.

3 Cid J, Carbassé G, Pereira A, Sanz C, Mazzara R, Escolar G, et al. Platelet transfusions from $\mathrm{D}+$ donors to $\mathrm{D}$ - patients: a 10 -year follow-up study of 1014 patients. Transfusion. 2011 Jun; 51(6):1163-9.

4 Cid J, Yazer MH, Lozano M. Platelet transfusion and respecting patient D type. Curr Opin Hematol. 2015 Nov;22(6):540-6.

5 Jakobowicz R, Williams L, Silberman F. Immunization of Rh-negative volunteers by repeated injections of very small amounts of Rh-positive blood. Vox Sang. 1972;23(4): 376-81.

6 Atoyebi W, Mundy N, Croxton T, Littlewood TJ, Murphy MF. Is it necessary to administer anti-D to prevent RhD immunization after the transfusion of $\mathrm{RhD}$-positive platelet concentrates? Br J Haematol. 2000 Dec;111(3): 980-3.

7 Solh Z, Athale U, Arnold DM, Cook RJ, Foley R, Heddle NM. Transfusion-related alloimmunization in children: epidemiology and effects of chemotherapy. Vox Sang. 2016 Oct; 111(3):299-307.

8 Cid J, Lozano M, Ziman A, West KA, O’Brien KL, Murphy MF, et al.; Biomedical Excellence for Safer Transfusion collaborative. Low frequency of anti-D alloimmunization following D+ platelet transfusion: the Anti-D Alloimmunization after D-incompatible Platelet Transfusions (ADAPT) study. Br J Haematol. 2015 Feb;168(4):598-603.

9 Reckhaus J, Jutzi M, Fontana S, Bacher VU, Vogt M, Daslakis M, et al. Platelet Transfusion Induces Alloimmunization to $\mathrm{D}$ and Non-D Rhesus Antigens. Transfus Med Hemother. 2018 May;45(3):167-72.

10 Seheult JN, Triulzi DJ, Yazer MH. I am the 9\%: making the case for whole-blood platelets. Transfus Med. 2016 Jun;26(3):177-85.

11 Menitove JE. Immunoprophylaxis for D- patients receiving platelet transfusions from $\mathrm{D}$ donors? Transfusion. 2002;42:136-8. Transfusion. 2002 Dec;42(12):1618.
12 Cid J, Ortin X, Elies E, Castellà D, Panadés M, Martín-Vega C. Absence of anti-D alloimmunization in hematologic patients after D-incompatible platelet transfusions. Transfusion. 2002 Feb;42(2):173-6.

13 Thibault L, Beauséjour A, Jacques A, Ducas E, Tremblay M. Overnight storage of whole blood: cooling and transporting blood at room temperature under extreme temperature conditions. Vox Sang. 2014 Feb;106(2): 127-36.

14 Schmidt M, Spengler HP, Lambrecht B, Hourfar MK, Seifried E, Tonn T. A new oneplatform flow cytometric method for residual cell counting in platelet concentrates. Transfusion. 2009 Dec;49(12):2604-11.

15 Santana JM, Dumont LJ. A flow cytometric method for detection and enumeration of low-level, residual red blood cells in platelets and mononuclear cell products. Transfusion. 2006 Jun;46(6):966-72.

16 Baumgarth N, Roederer M. A practical approach to multicolor flow cytometry for immunophenotyping. J Immunol Methods. 2000 Sep;243(1-2):77-97.

17 Lichtiger B, Surgeon J, Rhorer S. Rh-incompatible platelet transfusion therapy in cancer patients. A study of 30 cases. Vox Sang. 1983; 45(2):139-43

18 Duboeuf S, Flourié F, Courbil R, Oriol P, Benamara A, Chartier M, et al. [Anti-RH1 (anti-D) alloimmunization after transfusion of platelet concentrates from RH :1 donors]. Ann Biol Clin (Paris). 2009 May-Jun;67(3): 353-7.

19 Beckers EA, Faas BH, Ligthart P, Overbeeke $\mathrm{MA}$, von dem Borne AE, van der Schoot CE, et al. Lower antigen site density and weak D immunogenicity cannot be explained by structural genomic abnormalities or regulatory defects of the RHD gene. Transfusion. 1997 Jun;37(6):616-23.

20 Cid J, Lozano M. Risk of Rh(D) alloimmunization after transfusion of platelets from $\mathrm{D}+$ donors to D- recipients. Transfusion. 2005 Mar;45(3):453.

21 Frohn C, Dümbgen L, Brand JM, Görg S, Luhm J, Kirchner H. Probability of anti-D development in $\mathrm{D}$ - patients receiving $\mathrm{D}+\mathrm{RBCs}$. Transfusion. 2003 Jul;43(7):893-8.

22 Desai PC, Deal AM, Pfaff ER, Qaqish B, Hebden LM, Park YA, et al. Alloimmunization is associated with older age of transfused red blood cells in sickle cell disease. Am J Hematol. 2015 Aug;90(8):691-5.

23 Curtis BR. Recent progress in understanding the pathogenesis of fetal and neonatal alloimmune thrombocytopenia. Br J Haematol. 2015 Dec;171(5):671-82
24 Ryder AB, Zimring JC, Hendrickson JE. Factors Influencing RBC Alloimmunization: Lessons Learned from Murine Models. Transfus Med Hemother. 2014 Nov;41(6):406-19.

25 Fasano RM, Booth GS, Miles M, Du L, Koyama T, Meier ER, et al. Red blood cell alloimmunization is influenced by recipient inflammatory state at time of transfusion in patients with sickle cell disease. Br J Haematol. 2015 Jan; 168(2):291-300.

26 Hendrickson JE, Tormey CA. Understanding red blood cell alloimmunization triggers. $\mathrm{He}$ matology (Am Soc Hematol Educ Program). 2016 Dec;2016(1):446-51.

27 Piccin A, Murphy WG, Smith OP. Circulating microparticles: pathophysiology and clinical implications. Blood Rev. 2007 May;21(3): 157-71.

28 Westhoff CM. The structure and function of the Rh antigen complex. Semin Hematol. 2007 Jan;44(1):42-50.

29 Kumpel B. Are weak D RBCs really immunogenic? Transfusion. 2006 Jun;46(6):1061-2; discussion 1062-6.

30 Canellini G, Rubin O, Delobel J, Crettaz D, Lion N, Tissot JD. Red blood cell microparticles and blood group antigens: an analysis by flow cytometry. Blood Transfus. 2012 May; 10 Suppl 2:s39-45.

31 Tissot JD, Rubin O, Canellini G. Analysis and clinical relevance of microparticles from red blood cells. Curr Opin Hematol. 2010 Nov; 17(6):571-7.

32 Simak J, Gelderman MP. Cell membrane microparticles in blood and blood products: potentially pathogenic agents and diagnostic markers. Transfus Med Rev. 2006 Jan;20(1): $1-26$.

33 Victoria EJ, Branks MJ, Masouredis SP. Immunoreactivity of the Rho(D) antigen in cytoskeleton-free vesicles. Transfusion. 1987 Jan-Feb;27(1):32-5

34 Gelderman MP, Simak J. Flow cytometric analysis of cell membrane microparticles. Methods Mol Biol. 2008;484:79-93.

35 Oreskovic RT, Dumaswala UJ, Greenwalt TJ. Expression of blood group antigens on red cell microvesicles. Transfusion. 1992 NovDec;32(9):848-9.

36 Jy W, Ricci M, Shariatmadar S, Gomez-Marin O, Horstman LH, Ahn YS. Microparticles in stored red blood cells as potential mediators of transfusion complications. Transfusion. 2011 Apr;51(4):886-93. 